\title{
A Parameters Selection Method of SVM
}

\author{
Deqi KOU, Yuan ZHANG, Hanyue ZHENG \\ Department of Technical Support Engineering \\ Academy of Armored Forces Engineering \\ Beijing, China \\ niyuange1126@126.com
}

\begin{abstract}
An improved artificial fish swarm algorithm called ASFSA is proposed. It could facilitate the selection of values for Step and Visual to meet the balance of algorithm speed and effect. A new SVM parameters selection method based on the ASFSA is described, and the kernel parameter $\gamma$ and regularization parameter $C$ can both be optimized well. The application case shows that the performance of SVM with optimized parameters is good, so the method is feasible and effective.
\end{abstract}

Keywords-support vector machines;artificial fish swarm algorithm; parameters selection; son fish

\section{INTRODUCTION}

$\mathrm{SVM}^{[1-2]}$ (Support vector machines) is a machine learning algorithm based on the statistical learning theory which could cope well with small sample, nonlinear data and many other common problems. It is widely researched within the field of artificial intelligence ${ }^{[3]}$. Although the performance of SVM mostly depends on the selection of parameters and kernel, how to select parameters has no mature mathematical basis.

In order to solve the problem, the paper presents a parameters selection method of SVM based on an improved artificial fish swarm algorithm.

\section{SUPPORT VECTOR MACHINES}

The main idea of SVM is to make complex data $\mathrm{x}$ chaos to a high-dimension feature space $\mathrm{F}$ by a nonlinear chaotic $\varphi$ to make a linear regression. Once done, data classification is much easier. below:

A data integration $\mathrm{G}$ could be regressed by the equality

$$
f(x)=<\omega, \phi(x)>+b
$$

The parameter $\omega$ and $b$ in (1) are both regression factors which come from normalization and functional risk minimization:

$$
R(\omega)=\frac{1}{2}|\omega|^{2}+C \sum_{i=1}^{m} L_{\varepsilon}\left(f\left(x_{i}\right), y_{i}\right)
$$

$\mathrm{C}$ is the regularization parameter and $\mathrm{L}$ is the lost function. And usually:

$$
L(f(x), y)=\max \{|f(x)-y|-\varepsilon, 0\}
$$

Minimize $\mathrm{R}(\omega)$, and then

$$
\omega=\sum_{i=1}^{m}\left(\alpha_{i}-\alpha_{i}^{*}\right) \phi\left(x_{i}\right)
$$

Take $\omega$ into (1),

$$
\begin{aligned}
f(x) & =\sum_{i=1}^{m}\left(\alpha_{i}-\alpha_{i}^{*}\right)<\phi\left(x_{i}\right), \phi(x)>+b \\
& =\sum_{i=1}^{m}\left(\alpha_{i}-\alpha_{i}^{*}\right) k\left(x_{i}, x\right)+b
\end{aligned}
$$

And in (5),

$$
K\left(x_{i}, x\right)=<\phi\left(x_{i}\right), \phi(x)>
$$

$K\left(x_{i}, x\right)$, a positive index number, is the kernel of SVM , and it meets the Mercer Conditions. Some common kernels presents in Table 1.

$$
\text { TABLE I. TABLE } 1 \text { SOME COMMON KERNELS }{ }^{[4]}
$$

\begin{tabular}{|c|c|}
\hline Type & Mathematical Equation \\
\hline Linear & $K(x, y)=<x, y>$ \\
\hline Poly & $K(x, y)=(<x, y>+1)^{d}$ \\
\hline RBF & $K(x, y)=\exp (-\gamma<x-y, x-y>)^{2}$ \\
\hline STF & $K(x, y)=\max (1-g|<x-y, x-y>|, 0)$ \\
\hline CF & $K(x, y)=1 /\left(1+\gamma<x-y, x-y>^{2}\right)$ \\
\hline
\end{tabular}

When $\mathrm{b}$ is a point on edge, we could get the answer by KKT conditions, and we usually collect the average of the point on edge because of the stability:

$$
b=\operatorname{average}_{k}\left\{\delta_{k}+y_{k}-\sum_{i=1}^{m}\left(\alpha_{i}-\alpha_{i}^{*}\right) k\left(x_{1}, x_{k}\right)\right\}
$$

The mapping between entering space and feature space of SVM depends on the selection of parameters and kernel. Regularization parameter $\mathrm{C}$ is used to meet the balance between training errors and model complexity. But actually, $\mathrm{C}$ does not have a real meaning, so it is difficult to set a value.

How to set up the best model of the particular data has no perfect theory basis till now. Parameters are always set trying numbers, so there may be much error. Nowadays, research on kernel does get some results. It seems that the RBF kernel has a better performance, especially when we do not know regular pattern of the data ${ }^{[5-6]}$. The paper presents a parameters selection method to set C of SVM and $\gamma$ of the RBF kernel.

\section{PARAMETERS SELECTION}

Make performance index MAE the objective function $f(C, \gamma)$. 


$$
f(C, \gamma)=M A E=\sum_{i=1}^{n}\left|y_{i}^{\prime}-y_{i}\right| / n
$$

$\mathrm{y}_{\mathrm{i}}{ }^{\prime}$ above is the testing output, and $\mathrm{y}$ is the real output. $\mathrm{n}$ is the total of groups, and $\mathrm{f}$ is the Average Error of testing samples.

The parameters selection method has 3 steps. Step 1 is section Sample Extraction based on the Bagging method. Step 2 is section Parameters Optimization based on an improved AFSA. Step 3 is section Parameters Selection.

\section{A. Sample Extraction}

Step 1 is based on a sample auto extraction method called Bagging $^{[7]}$ which is widely used in many algorithms such as Random Forest.

Extract $2 / 3$ of the sample randomly as an Auto Sample, and there is $1 / 3$ of the ordinary sample left. Data left in sample after every extraction are called OOB (Out of Bag), and they are used to test the predictive results.

We define the number of extraction $\mathrm{m}$, so there are $\mathrm{m}$ groups with same data, but different extract samples and OOB.

In the groups, extraction samples are the training samples during parameters optimization. On the other hand, OOB is used to make a comparison in order to get the best parameters of that extraction sample.

\section{B. Parameters Optimization}

Taking experience and model complexity into consideration, we define the range of the parameters as follows.

$C \in\left(0,10^{3}\right), \gamma \in(0,10)$

The optimization method is ASFSA, an improved artificial fish swarm algorithm.

\section{1) Artificial Fish Swarm Algorithm ${ }^{[8]}$}

Artificial fish swarm algorithm (AFSA), firstly proposed by Ph.D. Li Xiaolei, is an efficient optimization method based on a model of autonomous animals. It copies some of fish behaviors including preying food, following, and swarming. Because of its strong robustness, high convergence speed and high efficiency, AFSA is widely used in electronic system optimization, assembly optimization, continuity optimization, information searching and many other fields.

\section{2) $A S F S A$}

Step and Visual, two parameters must be set first, has a strong impact on performance of AFSA. C has so large a range (sometimes 0 to $10^{6}$ ) that it is difficult to set values. Optimal results are achieved with small values of Step and Visual, but the algorithm may be very slow. The converse is also true. In order to resolve the problem, ASFSA (Artificial Son Fish Swarm Algorithm) which shows advantages over the performance of AFSA is presented. Figure 1 shows the flow diagram of ASFSA.

This research was supported by PLA General Headquarters research project.

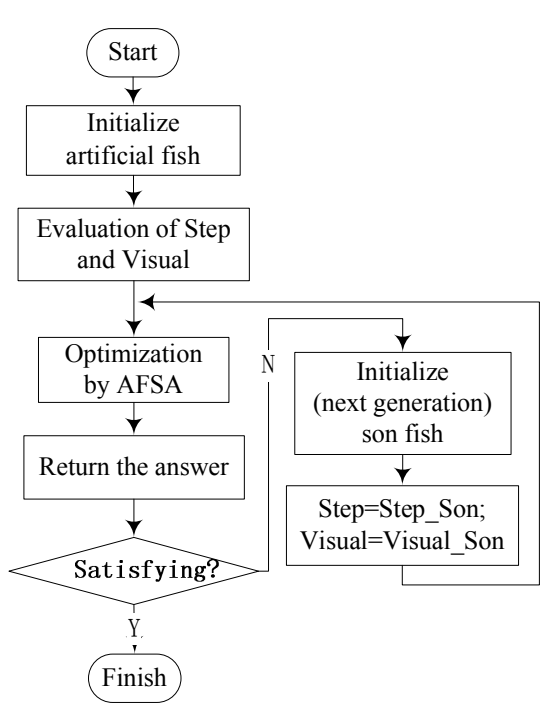

Figure 1. Flow diagram of ASFSA

Step and Visual are initially set as large values first, and we could get some function extreme points by AFSA with them. Although we could not get a satisfying value, the final value is in the range bounded by the extreme points.

Then release memory of the artificial fish, and initialize new ones with smaller Step and Visual. We call these fish son fish. Then, the son fish would make a more accurate optimization in the smaller range. If a higher precision is needed, we could create the $2^{\text {nd }}$ generation son fish, and maybe $3^{\text {rd }}$ or even the $\mathrm{n}^{\text {th }}$, and the Step and Visual would be smaller and smaller. When $f$ is one with a perfect precision we want, the next generation son fish would not be initialized while the current result would be returned.

Son fish is to make large mission smaller, so the searching range could turn to a smaller one divided by them the extreme points got from AFSA. It could avoid the insensible accurate searching within inappropriate ranges. We would get a precision value while the speed is also satisfying.

Make sure that the searching mission of son fish in falling generation is not as big as the one before. That means,

$$
\frac{\text { Step_Son }}{\text { Visual_Son }}>\frac{\text { Step }}{\text { Visual }}
$$

(9) would avoid the possibly much bigger astringent period.

We call AFSA with son fish AFSFA, an improved AFSA. It would meet a better balance of Step and Visual, so the precision and speed are both great.

3) Step and Visual

Typical values of Step and Visual rof artificial fish and son fish are given in Table 2.

TABLE II. STEP AND VISUAL

\begin{tabular}{|c|c|c|c|}
\hline Parameter & Artificial fish & $\begin{array}{c}\mathbf{1}^{\text {st }} \text { generation } \\
\text { son fish }\end{array}$ & $\begin{array}{c}\mathbf{2}^{\text {nd }} \text { generation } \\
\text { son fish }\end{array}$ \\
\hline Step & $(100,0.5)$ & $(10,0.1)$ & $(1,0.05)$ \\
\hline Visual & $(400,2)$ & $(30,0.2)$ & $(2,0.1)$ \\
\hline
\end{tabular}


As soon as satisfactory precision is achieved, subsequent generations of son fish are not initialized, reducing searching time

\section{PARAMETERS SELECTION}

ASFSA returns the local optimum coordinates accurately which are just the function extreme values. Then, it tries to seek for the perfect value among the extreme values which are the best values of every group, and we call them VIG (value in group) here. On the other hand, there are $m$ groups with $m$ VIGs after section Sample Extraction, and we should select the most satisfying one which is called VAG (value among groups) here.

VIGs are simply to compute that we just need to find the $f_{\min }$ from local minimums obtained by section Parameters Optimization. Make predictions of the whole sample with $m$ VIGs that we would get $m$ MAEs, and the minimum MAE is the VAG.

Sometimes, there may be two or more values with the best prediction precision, and we regard one with the minimum time complexity as the answer.

\section{AN APPLICATION CASE}

Libsvm, a SVM software programmed by Dr. Lin ChihJen, is widely used in research and engineering. The parameters selection method is implemented with $\mathrm{VC}++6.0$, and we will use Libsvm to see whether the parameters selection method of SVM is efficient.

The data in this case are 6 indexes to judge the engine technical states such as air cylinder pressure, engine acceleration, fuel consumption, vibration. We divide engine technical states into 4 categories including Excellent, Good, Normal and Broken. In order to reduce computation complexity brought by the oversized data, the 4 technical states are indicated by $(-1,1)$ forms as ones in Table 3 .

\section{TABLE III. TECHNICAL STATES IN CODING FORM}

\begin{tabular}{|l|c|c|c|c|}
\hline & Excellent & Good & Normal & Broken \\
\hline Coding & $(-1,-1)$ & $(-1,1)$ & $(1,-1)$ & $(1,1)$ \\
\hline
\end{tabular}

The sample size is 93 , divided it into two parts, a training sample with a capacity of 66 and a testing sample of 27 . The training sample is used to train SVM and also to set the parameters. The testing sample is used to make a prediction to verify. Table 4 shows details of the sample.

TABLE IV. DETAILS OF SAMPLE

\begin{tabular}{|c|c|c|c|c|c|}
\hline Type of sample & Capacity & $\mathbf{( - 1 , - 1 )}$ & $\mathbf{( - 1 , 1 )}$ & $\mathbf{( 1 , - 1 )}$ & $\mathbf{( 1 , 1 )}$ \\
\hline Original Sample & 93 & 77 & 11 & 3 & 2 \\
\hline Training sample & 66 & 56 & 7 & 2 & 1 \\
\hline Testing sample & 27 & 21 & 4 & 1 & 1 \\
\hline
\end{tabular}

For the training sample, we take 44 groups randomly as an auto sample, and 22 groups remained as OOB. The total frequency of sample extraction is 20 . For this case, VIG is the value corresponding to $\mathrm{MAE}_{\text {min }}$ in a certain extraction. Take VIGs into the $22 \mathrm{OOB}$, and the best VIG is the VAG. We define iteration times 50, and the section Parameters Selection stops working when $f<0.25$.

The result shows as follows.
VAG is $(128,0.35)$, and the other 2 of the best 3 VIGs are $(260,0.2)$ and $(202,0.05)$.

We define the 3 VIGs Para1, Para2 and Para3. Figure 3 shows the prediction error when they are taken into the testing sample.

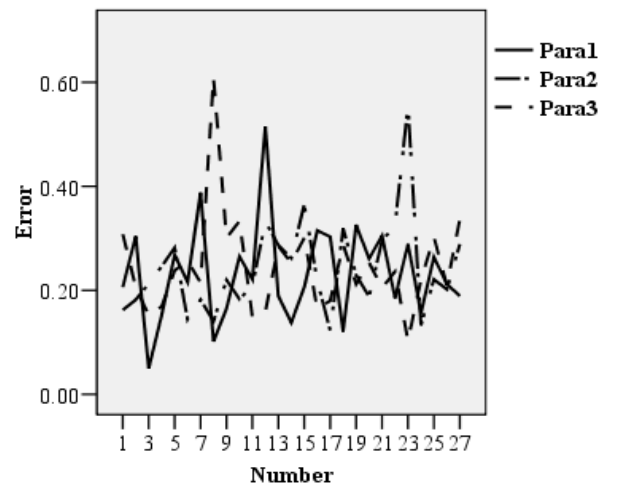

Figure 2. Prediction error

The 3 VIGs are compared with randomly chosen parameters in order to check out the superiority of the parameters selection method of SVM. Table 5 shows the result.

TABLE V. COMPARISON OF DIFFERENT PARAMETERS

\begin{tabular}{|c|c|c|c|}
\hline Parameters & $\begin{array}{c}\text { Generation } \\
\text { of son fish }\end{array}$ & Correct & Accuracy \\
\hline$(128,0.35)$ & $2^{\text {nd }}$ & 26 & $96.3 \%$ \\
\hline$(260,0.2)$ & $1^{\text {st }}$ & 26 & $96.3 \%$ \\
\hline$(202,0.05)$ & $2^{\text {nd }}$ & 26 & $96.3 \%$ \\
\hline$(10,0.2)$ & & 22 & $81.5 \%$ \\
\hline$(100,0.5)$ & & 25 & $92.7 \%$ \\
\hline$(200,0.3)$ & & 24 & $88.9 \%$ \\
\hline
\end{tabular}

Just as data in Table 5, the VIGs are much better than the random parameters, and that shows the parameters selected are good.

\section{CONCLUSION}

A parameter selection method of SVM based on AFSFA, an improved AFSA, has been presented. When building a SVM model, the kernel is always set as RBF, and the method could get the value by a MAE function $f(C, \gamma)$ of data in sample. So we could get the most appropriate parameters $C$ and $\gamma$ with the method according to a definite sample. The application case demonstrates that parameters selected by the method shows advantages to SVM, so it is feasible and efficient.

Although scholars have proposed some improved SVM such as LS-SVM, PSVM in recent years, the performance of them also mainly depends on parameters and kernels. And so, the parameters selection method is also suitable for them.

\section{REFERENCES}

[1] V N Vapnik, The nature of statistical learning theory, New York: Springer, 1995.

[2] V. N. Vapnik, The nature of statistical lerning, 2nd ed, New York: Springer, 2000.

[3] A D Sanchez, "Advanced support vector machines and kernel methods", Neurcomputing, vol. 55, pp. 5-20, January 2003. 
[4] Y. X. Chen and J. Z. Wang, "Support vector learning for fuzzy rule based classfication systems", IEEE Transactions of Fuzzy Systems, vol 11, pp.716-728, December 2003.

[5] S. S. Keethi and C. J. Lin, "Asymptotic behaviors of support vector machines with Gaussian kernel" Neural computation, vol 15, pp. 1667-1689, October 2003
[6] Y. Wang and B. G. Hu, "A Study on Integrated Evaluating Kernel Classification Performance Using Statistical Methods", Chinese journal of computers, vol 31, pp 942-952, June 2008.

[7] L. Breinan, "Bagging predictors", Machine learing, vol 24, pp. 123140, Febrary 1996.

[8] X L Li, Z J Shao and J X Qian, "An optimizing method based on autonomous animals: Fish - swarm algorithm", System engineering therry and pratice, vol 1, pp. 32-38, November 2002. 